ARTICLE

\title{
Contrasting epigenetic control of transgenes and endogenous genes promotes post-transcriptional transgene silencing in Arabidopsis
}

Nicolas Butel (1) 1,2,5, Agnès Yu ${ }^{1,5}$, Ivan Le Masson', Filipe Borges (10 1, Taline Elmayan1, Christelle Taochy 1,3, Nial R. Gursanscky (10 ${ }^{3}$, Jiangling $\mathrm{CaO}^{3}$, Shengnan Bi (ib ${ }^{3}$, Anne Sawyer ${ }^{3,4}$, Bernard J. Carroll (i) ${ }^{3,6 凶} \&$ Hervé Vaucheret ${ }^{1,6 \times}$

Transgenes that are stably expressed in plant genomes over many generations could be assumed to behave epigenetically the same as endogenous genes. Here, we report that whereas the histone H3K9me2 demethylase IBM1, but not the histone $\mathrm{H} 3 \mathrm{~K} 4 \mathrm{me} 3$ demethylase JMJ14, counteracts DNA methylation of Arabidopsis endogenous genes, JMJ14, but not IBM1, counteracts DNA methylation of expressed transgenes. Additionally, JMJ14mediated specific attenuation of transgene DNA methylation enhances the production of aberrant RNAs that readily induce systemic post-transcriptional transgene silencing (PTGS). Thus, the JMJ14 chromatin modifying complex maintains expressed transgenes in a probationary state of susceptibility to PTGS, suggesting that the host plant genome does not immediately accept expressed transgenes as being epigenetically the same as endogenous genes.

\footnotetext{
${ }^{1}$ Institut Jean-Pierre Bourgin, UMR 1318, INRAE, AgroParisTech, Université Paris-Saclay, Versailles, France. ${ }^{2}$ Université Paris-Sud, Université Paris-Saclay, Orsay, France. ${ }^{3}$ School of Chemistry and Molecular Biosciences, The University of Queensland, Brisbane, QLD, Australia. ${ }^{4}$ Queensland Alliance for Agriculture and Food Innovation, The University of Queensland, Brisbane, QLD, Australia. ${ }^{5}$ These authors contributed equally: Nicolas Butel, Agnès Yu.

${ }^{6}$ These authors jointly supervised this work: Bernard J. Carroll, Hervé Vaucheret. ${ }^{凶}$ email: b.carroll@uq.edu.au; herve.vaucheret@inrae.fr
} 
$\mathrm{R}$ NA-mediated gene silencing is a highly conserved eukaryotic mechanism that regulates endogenous gene expression and acts as a defense mechanism against viruses and transposons ${ }^{1-3}$. It occurs when double-stranded RNA (dsRNA) is formed by transcription of an inverted repeat, the copying of single-stranded RNA (ssRNA) by viral and host RNA-dependent RNA polymerases (RDRs), or the simultaneous transcription of both the positive and negative strands of DNA. DICER-like (DCL) proteins convert the dsRNA into microRNAs (miRNAs) or small-interfering RNAs (siRNAs), which then guide ARGONAUTE-like (AGO) proteins to mediate sequence-specific gene silencing.

In plants, 24 nucleotide (nt) siRNAs guide transcriptional gene silencing (TGS) and RNA-directed DNA methylation $(\operatorname{RdDM})^{4-6}$. The process of de novo RdDM also requires the cytosine methyltransferase DRM2 ${ }^{7}$. Following the establishment of DNA methylation and through subsequent rounds of DNA replication, cytosine methylation at $\mathrm{CG}$ and $\mathrm{CHG}$ sites can be maintained in the absence of siRNA by the cytosine methyltransferases MET1, and CMT2 and CMT3, respectively, whereas maintenance of $\mathrm{CHH}$ methylation requires the continual presence of $24 \mathrm{nt}$ siRNAs and DRM2 ${ }^{7,8}$.

Abundant siRNAs are also derived from viral RNA and RNA Polymerase II (Pol II) transcripts that have been converted into dsRNA by viral or host RDRs. In plants, RDR6 plays the major role in producing these dsRNAs, which are processed by DCL4 and DCL2 into $21 \mathrm{nt}$ and $22 \mathrm{nt}$ siRNAs, respectively. These siRNAs form a complex with AGO1 and guide silencing of complementary RNA viruses and post-transcriptional gene silencing (PTGS) of complementary mRNAs ${ }^{9}$. Once PTGS is triggered, the biogenesis of 21 and 22 nt siRNAs usually extends along the entire length of the mRNA in a process known as transitivity ${ }^{10-12}$. Furthermore, once induced, PTGS moves systemically throughout the plant $2,13,14$

It is well established that over-expression of aberrant mRNAs lacking a $5^{\prime}$ cap $^{15,16}$ or poly(A) tail ${ }^{17}$ induces RDR6-dependent PTGS in plants. The transcription of aberrant RNAs is a byproduct of RNA Pol II transcription in all eukaryotes, but normally, aberrant RNAs are intercepted and removed by the highly conserved RNA Quality Control (RQC) pathway. In addition to removing aberrant RNAs, the RQC pathway modulates gene expression via mRNA turnover. The mechanism of mRNA turnover has been studied in much more detail than the fate of aberrantly transcribed RNA, and the first step in mRNA decay is the removal of the polyA tail, followed by the removal of the $5^{\prime}$ cap by the decapping complex, which is composed of DCP1, DCP2 and VARICOSE (VCS) in plants ${ }^{18}$. Once the stabilizing $5^{\prime}$ and $3^{\prime}$ modifications have been removed from the mRNA, the exposed RNA is degraded $5^{\prime}-3^{\prime}$ by XRN exoribonucleases, and $3^{\prime}$ $5^{\prime}$ by the exosome in all eukaryotes ${ }^{19}$. In Arabidopsis, $d p c 1, d c p 2$, $v c s, x r n$, and exosome mutations that compromise RQC allow aberrant RNA to accumulate and result in spontaneous RDR6dependent PTGS of both transgenes ${ }^{15,16,20-24}$ and endogenous genes $^{21,25}$.

Mutants impaired in the histone H3K4me3 demethylase JMJ14 have been identified in two independent forward genetic screens for PTGS-deficient mutants 26,27 . Molecular analysis of jmj14 plants compared to wild-type (WT) plants revealed a decrease in transgene siRNA accumulation, a decrease in transgene $\mathrm{H} 3 \mathrm{~K} 4 \mathrm{~m} 3$ and an increase in CHG methylation at the transgene promoter 26,28 . Thusfar, DNA methylation has only been mechanistically implicated in TGS of promoters in most eukaryotic lineages 7 , inhibition of transcriptional elongation in fungi ${ }^{29}$, and most recently, inhibition of aberrant transcription from the gene body, i.e., the intragenic regions, of plants ${ }^{30,31}$ and mammalian embryonic stem cells ${ }^{32}$. Therefore, JMJ14 could link DNA methylation to aberrant RNAs and PTGS. However, the increase of transgene DNA methylation in jmj14 mutants contrasted the decreased methylation of $\mathrm{CHH}$ and $\mathrm{CHG}$ sites observed in two endogenous transposons in jmj14 mutants ${ }^{27}$, and resembled more the effect of $i b m 1$ mutations on endogenous sequences. IBM1 encodes a histone $\mathrm{H} 3 \mathrm{~K} 9 \mathrm{me} 2$ demethylase, and ibm 1 mutants have been reported to cause increased $\mathrm{CHG}$ and $\mathrm{CHH}$ methylation in the gene body of endogenous genes ${ }^{33-35}$. Therefore, the effect of $j m j 14$ and $i b m 1$ mutations on the epigenetic features of transgenic versus endogenous sequences required further investigation.

In this work, we perform genome-wide DNA methylation analysis of a jmj14 mutant and show that very few endogenous loci exhibit a change in DNA methylation in the mutant compared to wild type, and the DNA methylation changes in the jmj14 mutant are restricted to genomic sequences that normally exhibit highly variable DNA methylation levels ${ }^{36,37}$. In contrast, we detect increased methylation at all sequence contexts (CG, $\mathrm{CHG}, \mathrm{CHH}$ ) in transgenic sequences of the jmj14 mutant, but not the $i b m 1$ mutant. We also show that JMJ14 promotes and IBM1 prevents transgene PTGS, respectively, and that the susceptibility of transgenes to PTGS correlates with low levels of DNA methylation in the transgene and high levels of transgene aberrant RNA, indicating that JMJ14 in combination with other chromatin modifying proteins plays a crucial role in establishing and maintaining stably expressed transgenes in an epigenetic state that is distinct from endogenous genes.

\section{Results}

A genetic screen for impaired systemic PTGS identifies additional jmj14 alleles. Using the transgenic line $10027-3^{38}$, a genetic screen recovered three jmj14 mutants, \#38, \#90 and \#148, that showed defects in root-to-shoot PTGS transmission (Fig. 1A). The 10027-3 GFP reporter system involves a constitutive $p 35 S$ : GFP transgene linked to another transgene that drives root tipspecific expression of a GF hairpin RNA homologous to $G F P^{38}$. In 10027-3 WT plants, PTGS of GFP is initiated in the root apex during embryogenesis, and then as the seedlings germinate, silencing spreads into the shoot apex such that all true leaves that form show complete PTGS of GFP ${ }^{38}$ (Fig. 1A). The systemic spreading of PTGS in this GFP reporter system requires RDR6dependent amplification of dsRNA, using the constitutive GFP mRNA target as a template ${ }^{38}$. The three $10027-3$ jmj14 mutants were identified by candidate gene sequencing on a collection of EMS-induced 10027-3 mutants showing defects in systemic PTGS $^{39}$. Mutants \#38 and \#90 carry a nonsense and splice site mutation in JMJ14, respectively, and both mutants showed a lack of GFP silencing in the shoot apex but as the seedlings develop, PTGS of GFP spreads into the petiole and mid-vein of mature leaves (Fig. 1A). In contrast, mutant \#148 contains a missense mutation in JMJ14 and showed a complete loss of GFP silencing (Fig. 1A).

F1 plants from crosses between mutants \#38 and \#90 (Fig. 1B), and between mutants \#148 and \#38 (Fig. 1C) displayed a defect in systemic PTGS, thereby confirming that the jmj14 mutations were the causative genetic defect in these mutants. These three mutants further substantiated the role of JMJ14 in RDR6dependent PTGS ${ }^{26}$, and suggest the possible involvement of JMJ14 in systemic spreading of PTGS. Hereafter, the \#38, \#90 and \#148 mutants will be referred to as jmj14-5, jmj14-6 and jmj14-7, respectively (Fig. 1D).

JMJ14 promotes aberrant RNA-based RDR6-dependent PTGS. The results presented above suggest that JMJ14 plays a role in the systemic spreading of PTGS. However, they do not exclude that 

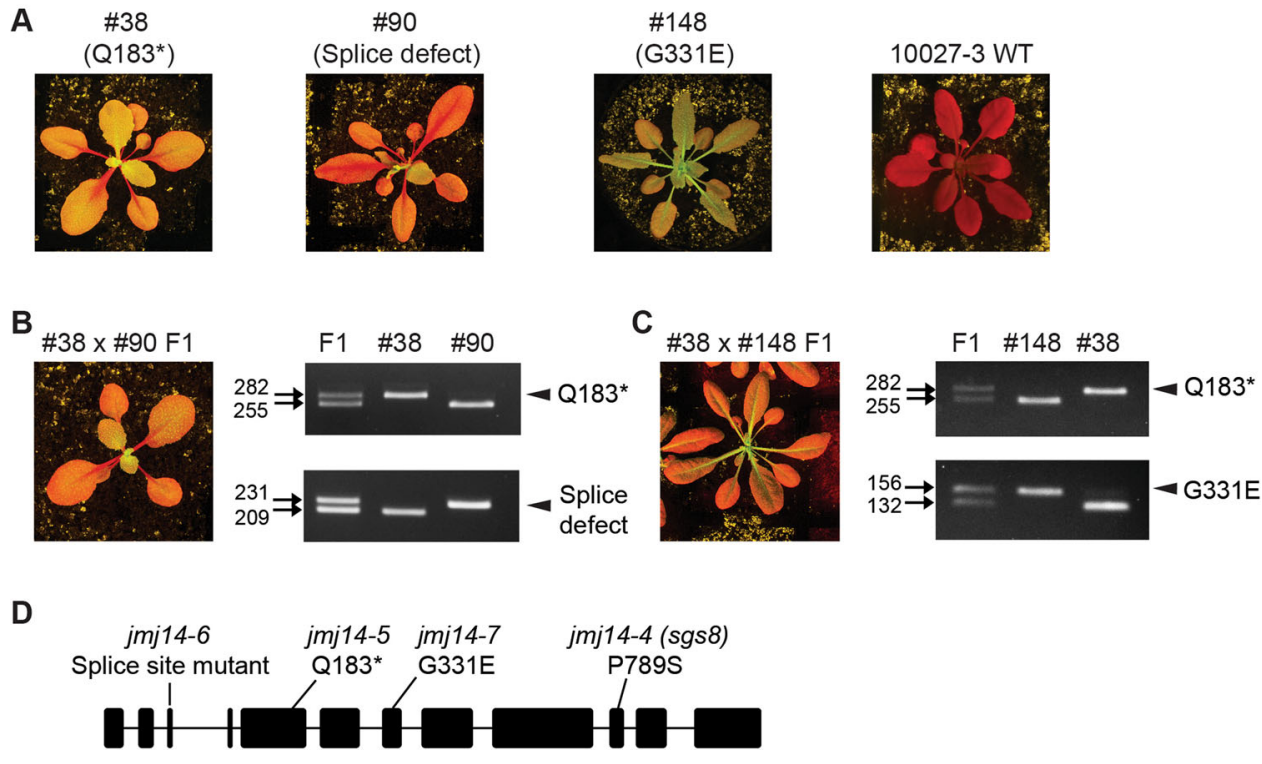

Fig. 1 Three new alleles of jmj14 show defects in systemic PTGS of GFP. A Rosette GFP phenotypes of EMS\#38 (Q183*; jmj14-5), EMS\#90 (splicing defect; jmj14-6) and EMS\#148 (G331E; jmj14-7). Transgenic line 10027-3 wild type (WT), the parent used in the genetic screen to identify jmj14-5, jmj14-6 and jmj14-7, shows systemic PTGS of GFP. B, C EMS\#90 (splice site mutant) and EMS\#148 (G331E), respectively, were not complemented by EMS\#38 (Q183*) as F1 plants from these crosses showed defective systemic PTGS. JMJ14 PCR genotyping assays are shown in the right-hand side of panel B, C; arrows indicate the size in bps of the larger jmj14 mutant allele and smaller WT allele for each PCR test and arrowheads indicate the diagnostic mutant jmj14 allele compared to respective WT allele. The results for one F1 plant from each cross are shown in the Figure, however, we confirmed the phenotype and genotype of at least four F1 plants from each cross. D Location of the new jmj14 mutations and the previously reported jmj14 allele, jmj14-4 (P789S) ${ }^{26}$ in the JMJ14 locus (AT4G20400). Exon and intron sequences are indicated by thick and narrow lines, respectively. Rosette images are of plants grown in soil under long-days for 4 weeks after planting. Uncropped and unprocessed scans of images of Fig. 1A-C are provided as a Source Data file.

JMJ14 also plays a role in the execution of PTGS. To resolve these two possibilities, the two-component GUS-silencing system $6 b 4$ 306 was used. This transgenic line consists of the p35S:GUS transgene locus called 664, which never triggers PTGS alone in WT plants, plus an unlinked $p 35 S: h p G U$ hairpin transgene locus called 306, which produces dsRNA and siRNAs homologous to the first half of the GUS coding sequence ${ }^{40}$. In contrast to the 10027-3 GFP reporter system, both the 664 GUS transgene and 306 the hairpin transgene are expressed constitutively, resulting in the execution of PTGS in every cell, and therefore does not require RDR6-dependent spreading of PTGS to silence the GUS transgene throughout the plant ${ }^{40}$. Crossing the 6b4-306 double transgenic line to the jmj14-4 mutant (P789S; Fig. 1D) produced triple homozygous 6b4-306 jmj14 plants in which PTGS occurred as efficiently as in 6b4-306 WT controls (Fig. 2A). Thus, JMJ14 is specifically required for RDR6-dependent PTGS, and not for the execution of PTGS when siRNAs are derived from a constitutively expressed hairpin transgene.

We next investigated the possibility that JMJ14 plays a role in the initiation of RDR6-dependent PTGS by enhancing the production of aberrant RNA from transgene loci. To address this question, we used the $6 b 4$ transgenic line that does not carry any additional T-DNA loci. As mentioned above, the 664 locus carries a $p 35 S$ :GUS transgene that never shows spontaneous PTGS in WT plants, but the introduction of $6 b 4$ in various RQCdeficient mutant backgrounds results in the spontaneous triggering of PTGS $16,21,22,24$. This result is explained by the $6 b 4$ GUS transgene producing low amounts of aberrant RNAs that are efficiently degraded by the cellular RQC pathways in WT plants. However, when RQC is impaired, aberrant RNAs derived from the 664 GUS transgene are converted into dsRNA by RDR6 and processed into 21- and $22 \mathrm{nt}$ siRNAs by DCL4 and DCL2, respectively, thus activating PTGS. To determine if JMJ14 was contributing to the production of aberrant RNA from the $6 b 4$
GUS transgene, we utilized the mRNA decapping-defective $v c s-9$ mutant to generate a $6 b 4$ vcs jmj14 double mutant, which was compared to $6 b 4 \mathrm{WT}, 664$ jmj 14 and $6 b 4$ vcs plants for the level of PTGS. The hypomorphic $v c s-9$ allele was used because $v c s-9$ mutants are viable and fertile, and because spontaneous PTGS occurs with $100 \%$ efficiency in $6 b 4 v c s-9$ plant $^{21}$. We observed an increase in GUS activity and a reduction in GUS siRNA accumulation in 664 vcs jmj14 compared to $6 b 4$ vcs plants (Fig. 2B). Given that JMJ14 is not involved in the production of siRNA derived from constitutively expressed hairpin RNA, these results strongly suggest that JMJ14 promotes PTGS by enhancing the production of aberrant RNA from the 664 GUS transgene.

JMJ14 impairment decreases the production of GUS aberrant RNAs. We previously identified an uncapped RNA, antisense to the GUS mRNA, hereafter referred to as aberrant SUG (abSUG) $\mathrm{RNA}^{11}$. Although it is not certain that this abSUG RNA is the aberrant RNA that triggers PTGS, its abundance correlates perfectly with the efficiency of PTGS in the two p35S:GUS reference lines $6 b 4$ and $L 1$. Indeed, it is detected at very low levels in 664 plants, which do not trigger PTGS spontaneously. It is also detected at only low levels in $664 \times r n 3$ xrn 4 and $L 1$ plants, which trigger PTGS spontaneously with $100 \%$ efficiency, most likely because the abSUG RNA is degraded by PTGS, similar to GUS mRNA. However, abSUG RNA accumulates to higher levels in $6 b 4 x r n 3 x r n 4 r d r 6$ and $L 1 r d r 6$ plants, but remains low in $6 b 4$ $r d r 6$ plants. Given that the $p 35 S$ : GUS transgene at the $L 1$ locus is transcribed at a higher level than its identical transgene counterpart at the 664 locus, these results suggest that the production of abSUG at a high level in $L 1$ plants or the absence of its degradation in $664 \times \mathrm{xrn} 3 \mathrm{xrn} 4$ plants could explain the capacity of these plants to trigger PTGS ${ }^{11}$.

To further test the hypothesis that jmj14 mutations limits the production of aberrant RNAs, the abundance of abSUG RNA was 
A

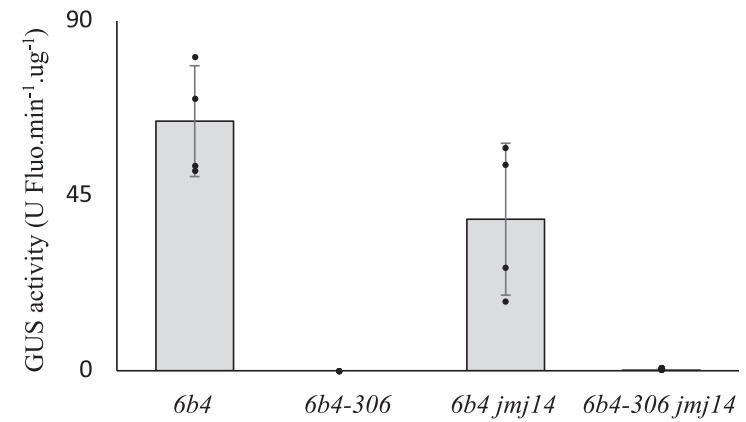

B
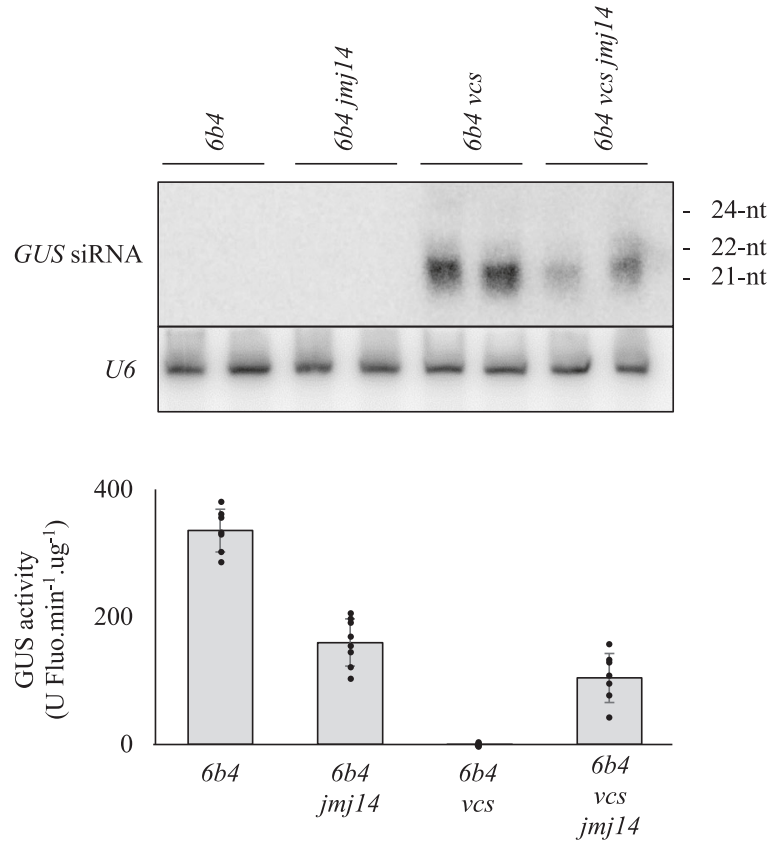

Fig. 2 JMJ14 promotes aberrant RNA-induced PTGS, but is not required for PTGS induced by hairpin dsRNA. A Effect of JMJ14 impairment on the transgenic $6 b 4$ GUS-expressing line and the 6b4-306 GUS-silenced line in which GUS mRNA produced by the $6 b 4$ locus is silenced by the consitutive expression of GUS hairpin dsRNA by the 306 locus. No PTGS is observed in $6 b 4$ and $6 b 4$ jmj14 plants, whereas PTGS occurs efficiently in both 6b4-306 and 6b4-306 jmj14 plants. Results represent the mean of four replicates. Errors bars represent the standard deviation of these four replicates. B Effect of JMJ14 impairment on the transgenic 6b4 GUS-expressing line and the $6 b 4$ vcs GUS-silenced line in which the $6 b 4$ GUS transgene undergoes aberrant RNA-induced PTGS due to the loss of RQC. PTGS occurs efficiently in $6 b 4$ vcs plants but is reduced in 664 vcs jmj14 plants, revealed by reduced accumulation of GUS siRNAs and increased GUS activity in $6 b 4$ vcs jmj14 compared to $6 b 4$ vcs plants. Results represent the mean of eight replicates. Errors bars represent the standard deviation of these eight replicates. Uncropped and unprocessed scans of images in Fig. 2B are provided as a Source Data file.

measured in L1, L1 rdr6 and L1 jmj14 rdr6 plants. To reliably assess the effect of JMJ14 on the levels of the abSUG RNA, the $r d r 6$ mutant background was used to avoid RDR6-dependent PTGS of the abSUG RNA. The amount of abSUG RNA was very low in L1 WT plants compared to L1 rdr6 plants, thereby confirming that abSUG RNA is degraded by PTGS in L1 WT plants (Fig. 3). However, the amount of abSUG RNA in the L1 rdr6 mutant was at least double the amount in the L1 jmj14 rdr6 double mutant (Fig. 3), which further supports the

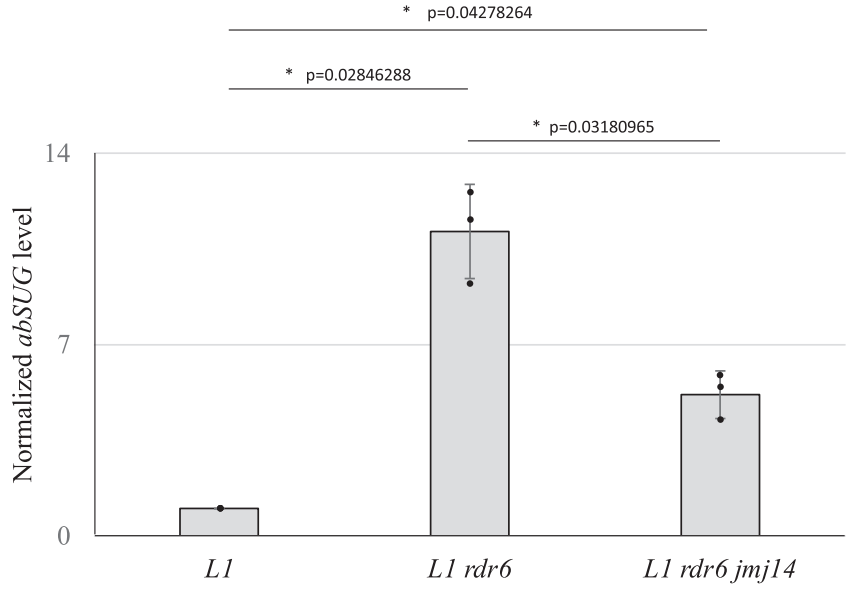

Fig. 3 Impairing JMJ14 decreases the steady state level of aberrant abSUG RNA. Normalized expression of abSUG RNA in $L 1, L 1 \mathrm{rdr} 6$ and $L 1 \mathrm{rdr} 6$ jmj14 plants. Results are expressed as a fold change compared to $L 1(L 1=1)$ and normalized to elF7a. Error bars represent the standard deviation of three replicates. The significance was assayed with a Bonferroni corrected $t$-test $(P<0.05)$.

hypothesis that JMJ14 promotes transgene-derived aberrant RNA production.

A synergistic relationship between systemic PTGS signals and aberrant RNA in the induction of RDR6-dependent PTGS. The results described above strongly suggest that JMJ14 promotes the production of aberrant RNA from transgene loci, and that JMJ14 also plays a role in systemic PTGS. To investigate the possibility of a collaborative relationship between aberrant RNA produced from transgene loci and systemic PTGS signals in the induction of RDR6-dependent PTGS, we conducted reciprocal grafting experiments using WT and jmj14 mutants as rootstocks and scions in our GFP and GUS reporter systems for grafttransmissible PTGS ${ }^{13,38}$.

When GFP-expressing 10027-3 jmj14-7 scions were grafted onto GFP-silenced 10027-3 WT rootstocks, graft-transmissible PTGS failed to be initiated (Fig. 4A), suggesting that the jmj14-7 mutation completely abolished the capacity of the scion to respond to the systemic PTGS signal transmitted from the 100273 WT roots. A feature of PTGS in plants is the predominance of $21 \mathrm{nt}$ siRNAs produced by DCL4, along with a much lower abundance of $22 \mathrm{nt}$ siRNA produced by DCL2. Nevertheless, DCL2 and its $22 \mathrm{nt}$ siRNA play a more important role in systemic PTGS than DCL4 ${ }^{38,41}$. Therefore, we also grafted 10027-3 jmj14-7 scions onto 10027-3 dcl4-5 rootstocks that produce predominantly DCL2-dependent $22 \mathrm{nt}$ siRNAs $^{38}$, and again, grafttransmissible PTGS failed to be initiated in the jmj14-7 scions. These results suggest that jmj14 scions are incapable of responding to either DCL4-dependent $21 \mathrm{nt}$ or DCL2dependent $22 \mathrm{nt}$ siRNAs as mobile silencing signals.

We also grafted GFP-expressing scions of transgenic line 214 (Supplementary Fig. 1) onto 10027-3 or 10027-3 jmj14 mutant lines as rootstocks ${ }^{13}$. Whereas GFP PTGS is efficiently transmitted from 10027-3 rootstocks to 214 scions, we observed a decrease in systemic transmission of PTGS when 214 scions were grafted onto 10027-3 jmj14 rootstocks (Fig. 4A).

These findings were confirmed using the jmj14-4 mutant allele in the $6 b 4$ GUS reporter background. Whereas grafting $6 b 4$ WT scions grafted onto L1 WT rootstocks triggered efficient GUS PTGS in $6 b 4$ scions, $6 b 4$ jmj14-4 scions grafted onto L1 WT rootstocks did not trigger PTGS (Fig. 4B, C). In addition, 664 jmj14-4 scions also failed to initiate PTGS when grafted onto 
A

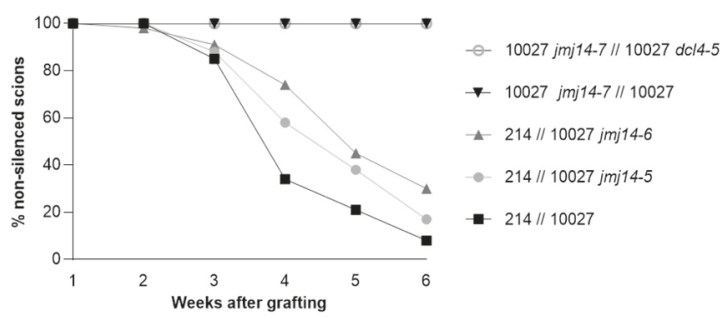

B

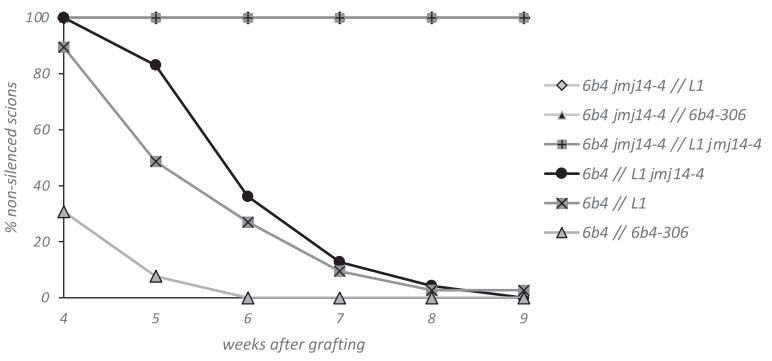

C

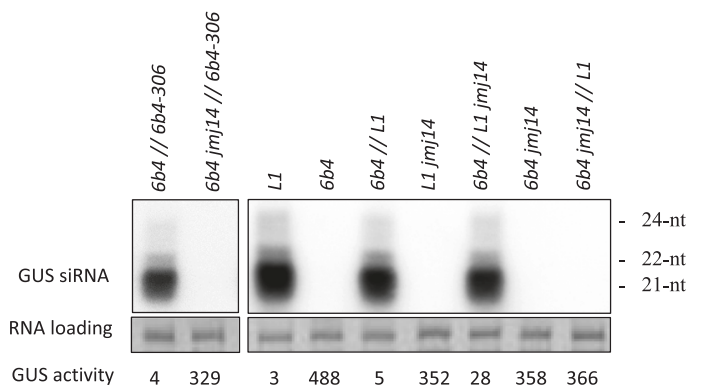

Fig. 4 JMJ14 is required for reception of graft-transmissible PTGS in scions and efficient transmission of PTGS from rootstocks. A Grafting results using the transgenic 214 GFP-expressing and 10027-3 GFP-silencing system for systemic PTGS. When GFP-expressing 10027-3 jmj14-7 scions were grafted onto either 10027-3 wild-type (WT) rootstocks that produce primarily DCL4-dependent $21 \mathrm{nt}$ siRNAs or 10027-3 dc/4-5 rootstocks producing predominantly DCL2-dependent $22 \mathrm{nt}$ siRNAs ${ }^{38}$, no systemic PTGS was observed. Similarly, when this most severe jmj14 allele, jmj14-7, was used as rootstocks and grafted to GFP-expressing 214 WT scions, no transmission of systemic PTGS was observed. Weaker alleles of $j m j 14$, i.e., jmj14-5 and jmj14-6, were also used as rootstocks grafted onto GFP-

expressing 214 WT scions ${ }^{38}$, and these mutant rootstocks showed delayed transmission of systemic PTGS compared to WT rootstocks. In total, 22 to 66 grafted plants were assessed over at least two independent experiments for each combination of grafted genotypes. B Grafting results using the transgenic GUS-expressing $6 b 4$ line and GUS-silencing 6b4-306 and $L 1$ lines as a system for systemic PTGS. When $6 b 4$ scions were grafted onto 6b4-306, L1 or L1 jmj14-4 rootstocks, systemic PTGS was observed, although delayed when using $L 1$ jmj14-4 rootstocks. In contrast, when $6 b 4$ jmj14-4 scions were grafted onto $6 b 4-306$ or $L 1$ rootstocks, no systemic PTGS was observed. In total, 16 to 74 grafted plants were assessed over at least two independent experiments for each combination of grafted genotypes. C GUS activity (units/ $\mu$ g protein/min) and GUS siRNA accumulation in ungrafted $6 b 4,6 b 4$ jmj14, L1, L1 jmj14 lines and $6 b 4 / / 6 b 4$ 306, 6 b4 jmj14 // 6b4-306, 6b4 // L1, 6b4 // L1 jmj14 and 6b4 jmj14 // L1 grafts. Each sample corresponds to a mix of four plants. The two blots were hybridized with the same probe and exposed the same amount of time. Uncropped and unprocessed scans of images in Fig. 4C are provided as a Source Data file. 6b4-306 rootstocks (Fig. 4C). Furthermore, similar to what was observed for jmj14-5 and jmj14-6 rootstocks using GFP as the reporter for PTGS (Fig. 4A), when GUS-expressing 664 WT scions were grafted onto $L 1$ jmj14-4 rootstocks, the onset of grafttransmissible PTGS in the scions was delayed (Fig. 4B). Altogether, these results indicate that JMJ14 plays an essential role in the perception of the PTGS systemic signal.

Transgene DNA methylation counteracts aberrant RNAinduced PTGS. Southern blot analysis using a methylationsensitive enzyme previously revealed that $L 1$ jmj14-3, L1 jmj14-4, $6 b 4$ jmj14-3, and $6 b 4$ jmj14-4 plants exhibit increased DNA methylation at CHG sites in the promoter of the p35S:GUS transgene ${ }^{26}$. To determine the methylation profile of the entire transgene locus in a jmj14 mutant background, whole-genome bisulfite sequencing (WGBS) analysis was performed on plants carrying the $6 b 4$ locus. WGBS revealed that the transgenic line $6 b 4$ carried a single T-DNA insertion. However, the left border did not delineate the $6 b 4$ T-DNA insertion and the entire binary vector was also found to have inserted adjacent to the left border (Supplementary Fig. 2). This phenomenon of co-transfer of nonT-DNA vector sequences occurs frequently with T-DNA integration events into plant chromosomes ${ }^{42}$. WGBS analysis revealed a high level of CG methylation in the GUS coding sequence of $6 b 4$ plants (Fig. 5), which most likely reflects the highly transcribed status of the p35S:GUS transgene in this transgenic line. Of note, almost no CG methylation was found in the adjacent NPTII transgene (Supplementary Fig. 3), consistent with a low level of NPTII expression and the $6 b 4$ line being barely resistant to kanamycin ${ }^{11}$. However, a surprisingly high level of CG methylation was observed in the non-T-DNA vector sequences inserted along with the T-DNA (Supplementary Fig. 3), suggesting that these sequences had been transcribed by a plant RNA polymerase prior to, and/or after integration into the chromosome. In contrast to $\mathrm{CG}$ methylation, the level of $\mathrm{CHH}$ and CHG methylation was very low in both T-DNA and vector sequences of the $6 b 4$ locus in the WT genetic background (Fig. 5 and Supplementary Fig. 3). The situation was very different in $6 b 4$ jmj14-4 plants where the GUS coding sequence exhibited CHG hypermethylation and the $35 S$ promoter showed increased $\mathrm{CHG}$ and $\mathrm{CHH}$ methylation (Fig. 5). Of note, $\mathrm{CHH}$ and CHG hypermethylation in $6 b 4$ jmj14 was not observed in the adjacent NPTII transgene but was observed in the vector sequences flanking the left border (Supplementary Fig. 3A), suggesting that $\mathrm{CHH}$ and $\mathrm{CHG}$ hypermethylation in jmj14 occurs mostly at sequences that show high levels of CG methylation in the WT genetic background.

These results prompted us to hypothesize that $\mathrm{CHH}$ and $\mathrm{CHG}$ hypermethylation of the p35S:GUS transgene in 6 b4 jmj14 plants could prevent, or at least reduce, the production of aberrant RNAs (Fig. 3), thus explaining why jmj14 scions cannot trigger PTGS upon grafting onto silenced rootstocks (Fig. 4). To test this hypothesis, $6 b 4 \mathrm{jmj} 14 \mathrm{drm} 2 \mathrm{cmt} 3$ plants were generated, and we confirmed the absence of $\mathrm{CHH}$ and $\mathrm{CHG}$ methylation in the p35s:GUS transgene and the entire $6 b 4$ locus in this line (Fig. 5 and Supplementary Fig. 3). When grafted onto GUS-silenced rootstocks, $6 b 4 j m j 14 \mathrm{drm} 2 \mathrm{~cm}$ t3 plants triggered PTGS as efficiently as grafted $6 b 4$ WT controls (Table 1), supporting the hypothesis that $\mathrm{CHH}$ and $\mathrm{CHG}$ hypermethylation is instrumental in preventing systemic PTGS in 664 jmj14 plants.

There is a low but detectable level of CHG methylation (but not $\mathrm{CHH}$ methylation) in the GUS coding sequence of $664 \mathrm{WT}$ plants (Fig. 5), and we asked if this residual level of $\mathrm{CHG}$ methylation prevents spontaneous induction of PTGS in these 
A

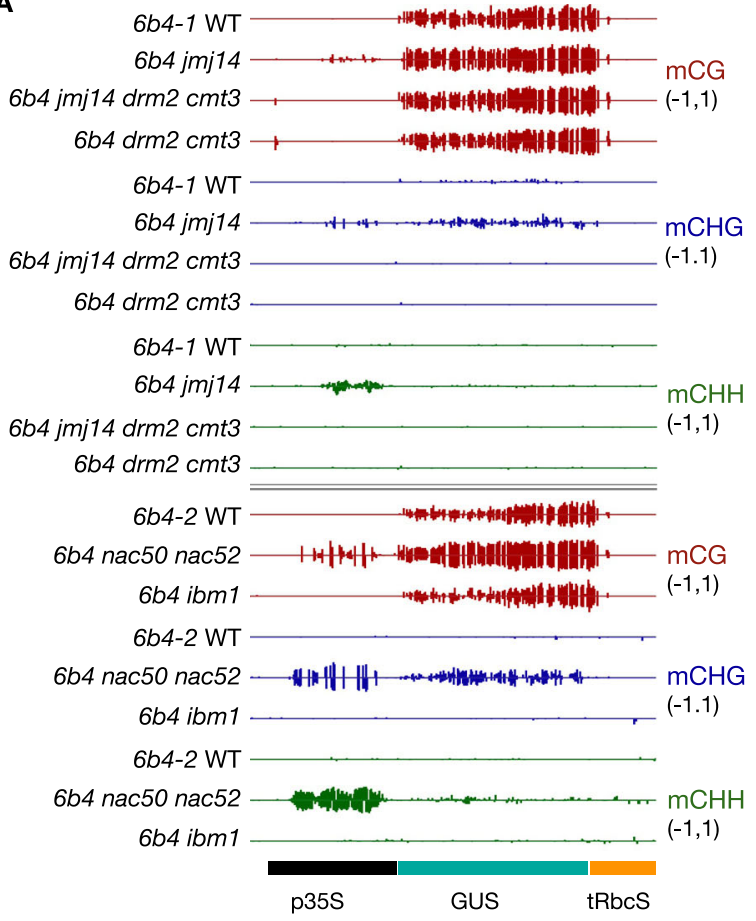

B
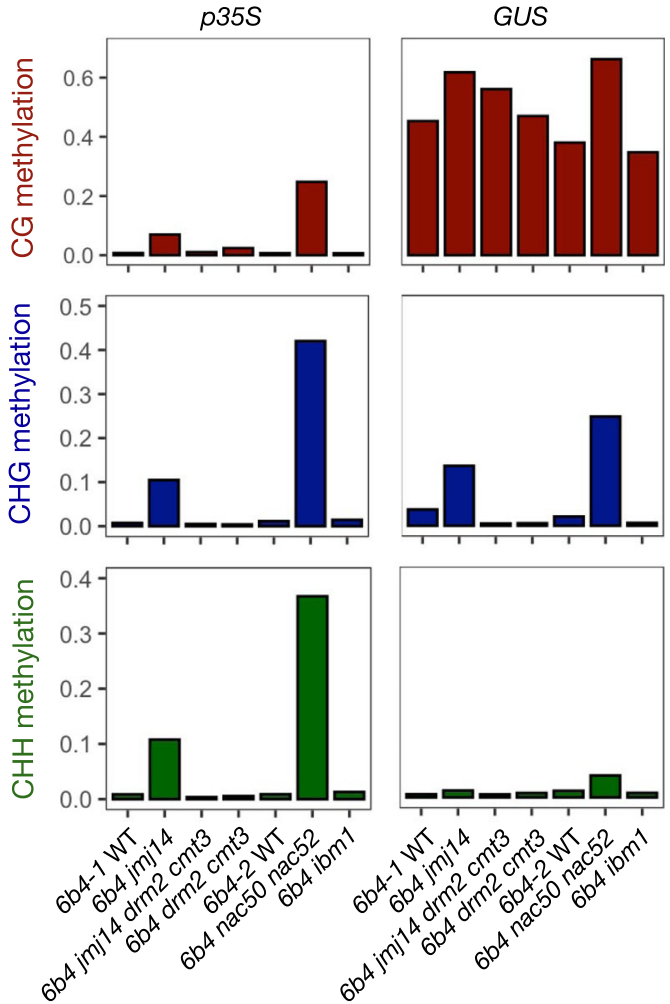

Fig. 5 JMJ14 and NAC50/NAC52 prevent CHG and CHH methylation of the p35S:GUS transgene. A CG, CHG and CHH methylation (where $\mathrm{H}=\mathrm{A}, \mathrm{C}$ or T) profiles for the 664 GUS transgene consisting of a modified $35 S$ promoter (p35S), the GUS coding sequence (GUS) and a Rubisco terminator (tRbcS). The top panel shows DNA methylation profiles of 5-week-old plants for $6 b 4$ WT control 1 (6b4-1 WT), and mutants $6 b 4$ jmj14, $6 b 4$ jmj14 drm2 cmt3 and $6 b 4$ $\mathrm{drm} 2 \mathrm{cmt3}$. The bottom panel shows DNA methylation profiles of 8-week-old plants for $6 \mathrm{~b} 4 \mathrm{WT}$ control 2 (6b4-2 WT) and mutants $6 \mathrm{~b} 4$ nac50 nac52 and $6 b 4 \mathrm{ibm1}$. Bisulfite data were analyzed using R version 4.0.2. B Proportion of methylated cytosines in the three sequence contexts across the $p 35 S$ and GUS sequences show that increased $\mathrm{CHG}$ and $\mathrm{CHH}$ methylation in $6 b 4$ jmj14 and 664 nac50 nac52 plants is dependent on the DRM2-CMT3 pathway, and that impairment of IBM1, if anything, decreases rather than increases DNA methylation of the GUS coding sequence.

Table 1 Frequency of spontaneous and graft-induced PTGS.

\begin{tabular}{llllll} 
Genotype & Long-day & \% spontaneous PTGS & Short-day & \% spontaneous PTGS & \% graft-induced PTGS \\
\hline $6 b 4$ nac50 nac52 & BR1 & $0(n=48)$ & BR1 & $0(n=6)$ & $0(n=24)$ \\
& BR2 & $0(n=48)$ & BR2 & $0(n=6)$ & $0(n=24)$ \\
$6 b 4 j m j 14$ & BR1 & $0(n=48)$ & BR1 & $0(n=16)$ & $0(n=13)$ \\
& BR2 & $0(n=48)$ & BR2 & $0(n=16)$ & $0(n=27)$ \\
$6 b 4$ WT & BR1 & $0(n=48)$ & BR1 & $0(n=39)$ & $100(n=36)$ \\
& BR2 & $0(n=48)$ & BR2 & $0(n=32)$ & $100(n=48)$ \\
$6 b 4 j m j 14 c m t 3 d r m 2$ & BR1 & $0(n=48)$ & BR1 & $0(n=26)$ & $96(n=26)$ \\
$6 b 4 \mathrm{cmt3} d r m 2$ & BR2 & $0(n=48)$ & BR2 & $0(n=30)$ & $100(n=26)$ \\
& BR1 & $12(n=48)$ & BR1 & $9(n=11)$ & $100(n=8)$ \\
$6 b 4 i b m 1$ & BR2 & $8(n=48)$ & BR2 & $12(n=8)$ & nd \\
& BR1 & $19(n=48)$ & BR1 & nd & nd \\
\hline
\end{tabular}

Note: For each genotype tested, the frequency of spontaneous PTGS was first determined in long-day conditions. Two independent experiments were performed, each consisting of 48 plants. For grafting experiments, plants were grown in short-day conditions. Two independent experiments were performed for each grafting combination. In each experiment, non-grafted plants were kept as controls and analyzed to determine the frequency of spontaneous PTGS in short-day conditions. Plants were considered silenced when exhibiting <20 fluorescent GUS activity units per minute per microgram of total protein.

BR biological replicate, nd not determined.

plants. To test this hypothesis, $6 b 4 \mathrm{drm} 2 \mathrm{cmt} 3$ plants were generated. Our analysis revealed that $\mathrm{CHG}$ methylation is reduced in the GUS coding sequence of $6 b 4 \mathrm{drm} 2 \mathrm{cmt} 3$ plants compared to $664 \mathrm{WT}$ plants (Fig. $5 \mathrm{~A}$ ), and indeed, $\sim 10 \%$ of these plants trigger spontaneous PTGS (Table 1). These results strongly support the hypothesis that transgene CHG methylation strongly limits the production of aberrant RNAs that would otherwise induce spontaneous PTGS of the homologous GUS coding sequence. In $6 b 4 \mathrm{drm} 2 \mathrm{cmt} 3$ plants, we propose that the lower level of CHG methylation of the GUS coding sequence increases the transcription of aberrant RNAs, thereby allowing spontaneous PTGS to occur. While $6 b 4 \mathrm{WT}$ plants produce insufficient aberrant RNAs to trigger spontaneous PTGS of the GUS coding sequence, it is sufficient for graft-induced PTGS to occur due to a synergistic relationship between aberrant RNA expressed from the GUS transgene of $6 b 4$ WT scions and systemic PTGS signals 


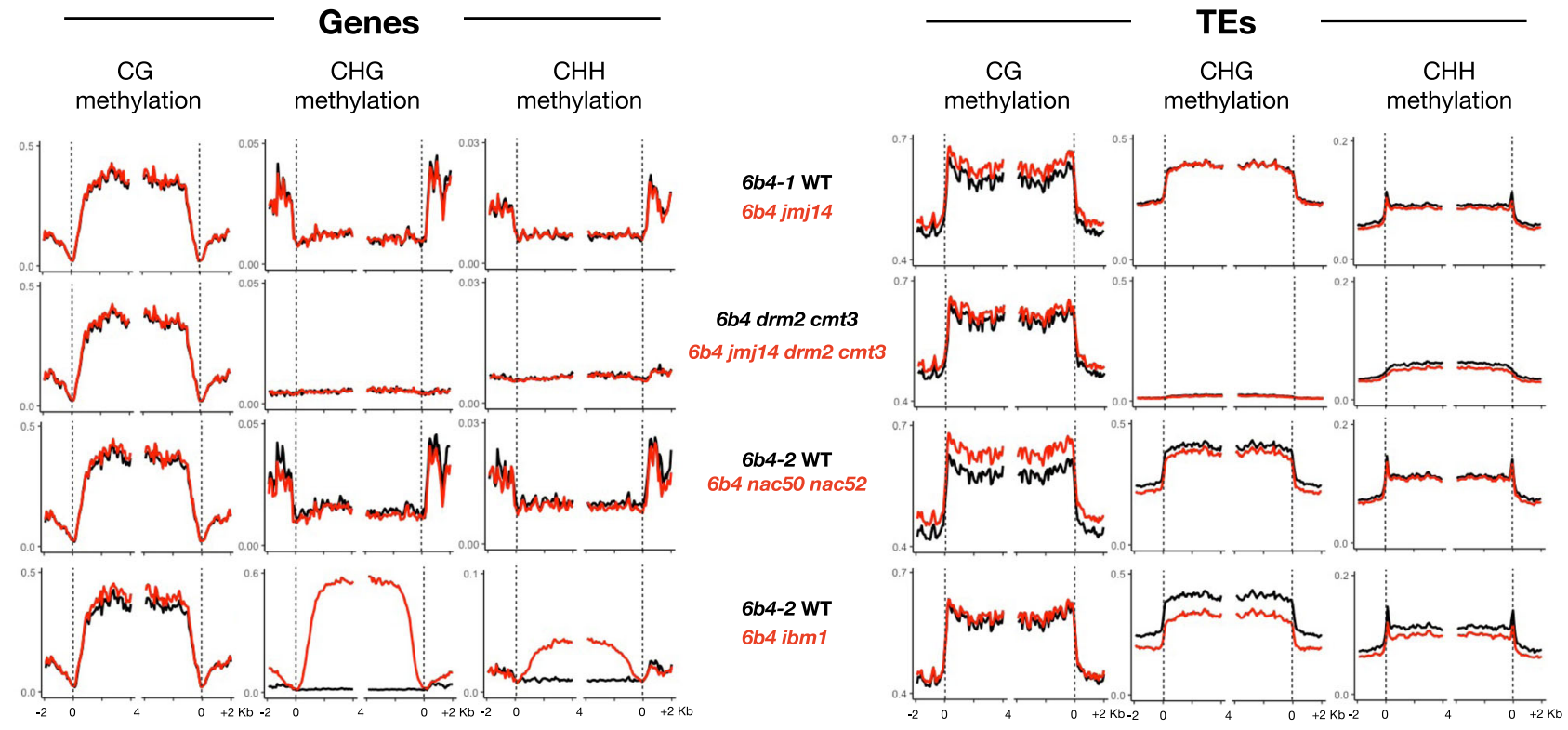

Fig. 6 JMJ14 and NAC50/NAC52 do not affect CHG and CHH methylation of endogenous genes and TEs. The gene body of endogenous genes (left panels) and TEs (right panels) were annotated according to the TAIR10 reference genome and aligned at the $5^{\prime}$ and $3^{\prime}$ ends (dashed lines), and average CG, CHG and CHH methylation levels for 100-bp intervals were plotted in pairwise comparisons between $6 b 4-1 \mathrm{WT}$ and $6 \mathrm{~b} 4 \mathrm{jmj} 14$, and $6 \mathrm{~b} 4 \mathrm{drm} 2 \mathrm{cmt} 3$ and $6 b 4$ jmj14 drm $2 \mathrm{cmt} 3$ of 5-week-old plants, and 6b4-2 WT and $6 b 4$ nac50 nac52, and 6b4-2 WT and $6 b 4$ ibm1 for 8-week-old plants. This analysis showed that $\mathrm{CHG}$ and $\mathrm{CHH}$ methylation in the body of endogenous genes and TEs is not affected in $664 \mathrm{jmj} 14$ and $6 b 4$ nac50 nac52 plants, whereas $\mathrm{CHG}$ and $\mathrm{CHH}$ is substantially increased in the body of endogenous genes in $6 b 4 \mathrm{ibm1}$ plants. Bisulfite data were analyzed using R version 4.0.2.

derived from GUS-silenced rootstocks. In contrast, in 664 jmj14 scions, increased CHG methylation further limits the production of aberrant RNA from the $6 b 4$ GUS transgene, rendering it nonresponsive to graft-transmissible PTGS signals from silenced GUS rootstocks.

Remarkably, WGBS of $6 b 4 \mathrm{WT}$ and $6 b 4$ jmj14-4 plants revealed that loss of JMJ14 had a limited effect on the extent of DNA methylation at endogenous loci (Fig. 6). A summary of all endogenous DMRs identified between 664 WT and $6 b 4$ jmj $14-4$ is presented in Supplementary Data 1. Only one endogenous hyper CHG DMR was identified in jmj14, and this endogenous region did not show hyper $\mathrm{CG}$ and hyper $\mathrm{CHH}$ methylation, whereas the transgenic sequences (both the T-DNA and the co-integrated backbone sequences of the binary vector) exhibited hyper CG, hyper $\mathrm{CHG}$ and hyper $\mathrm{CHH}$ methylation (Fig. 5 and Supplementary Fig. 3).

Both JMJ14 and NAC52 interact with the p35S:GUS transgene and are required for systemic PTGS. Mutations in JMJ14 were previously shown to adversely affect Pol II occupancy and H3K4me3 levels in the promoter of the p35S:GUS transgene in transgenic line $6 b 4^{26}$. However, it was not determined if this effect was due to a direct interaction between JMJ14 and chromatin at the $35 S$ promoter. To address this question, chromatin immunoprecipitation (ChIP) of JMJ14 was performed on the 664 jmj14-4 line complemented with a pJMJ14:3xFlag-JMJ14 transgene ${ }^{43}$. ChIP was conducted using Flag antibodies and followed by quantitative PCR (qPCR) using primer pairs located in the $35 S$ promoter, and in the $5^{\prime}$ and $3^{\prime}$ regions within the GUS transgene body. Quantitative ChIP-PCR revealed a significant enrichment of JMJ14 primarily at the $35 S$ promoter (Fig. 7A). These results indicate that JMJ14 is a component of chromatin at the promoter of the $p 35 S: G U S$ transgene, and are consistent with Pol II occupancy, high levels of H3K4me $3^{26}$ and lack of DNA methylation (Fig. 5) within the $35 \mathrm{~S}$ promoter of 664 WT plants.
JMJ14 has also been shown to interact with the NAC-domain transcription factor NAC52 and its close relative NAC50 $0^{44,45}$. NAC50 and NAC52 most likely play redundant roles, but NAC52 appears more important than NAC50 in Arabidopsis due to its higher level of expression. Indeed, a genetic screen for PTGS impaired mutants identified a mutation, originally named sgs 1 , which turned out to be a mutant allele of NAC52, whereas reverse genetics showed that a nac50 knockout mutation had no effect on PTGS $^{28}$. To determine whether NAC50 or NAC52 participates in graft-induced PTGS of the 664 GUS transgene, a 664 nac50 nac52 double mutant was generated and grafted onto GUS-silenced rootstocks. Similar to $664 \mathrm{jmj} 14$, the 664 nac50 nac52 scions were incapable of triggering PTGS of GUS when grafted onto GUSsilenced rootstocks (Table 1), indicating that both JMJ14 and NAC50/NAC52 are required for reception of graft-induced PTGS of the 664 GUS transgene. In addition, WGBS analysis of nongrafted 664 nac50 nac52 plants revealed transgene $\mathrm{CHH}$ and CHG hypermethylation similar to non-grafted $6 b 4$ jmj14 plants (Fig. 5B and Supplementary Fig. 3B), confirming the correlation between $\mathrm{CHH}$ and $\mathrm{CHG}$ hypermethylation and decreased induction of PTGS.

Importantly, WGBS of 664 WT and 664 nac50 nac52 plants also confirmed that like the loss of JMJ14, loss of both NAC50 and NAC52 had a limited effect on the extent of DNA methylation at endogenous loci. Indeed, only one endogenous hyper CHG DMR was identified in jmj14 and only one endogenous hyper CHG DMR was identified in nac50 nac52 (Supplementary Data 1). These two DMRs were located on chromosome 5 and chromosome 3 , respectively, indicating that jmj14- and nac50 nac52-dependent hyper CHG methylation is only found in the transgenic sequences (both the T-DNA and the co-integrated backbone sequences of the binary vector). Moreover, transgenic sequences not only exhibited hyper CHG methylation but also hyper CG and hyper $\mathrm{CHH}$ methylation (Fig. 5 and Supplementary Fig. 3). The only endogenous hyper CHG methylation found in jmj14 did not show hyper CG and 
A

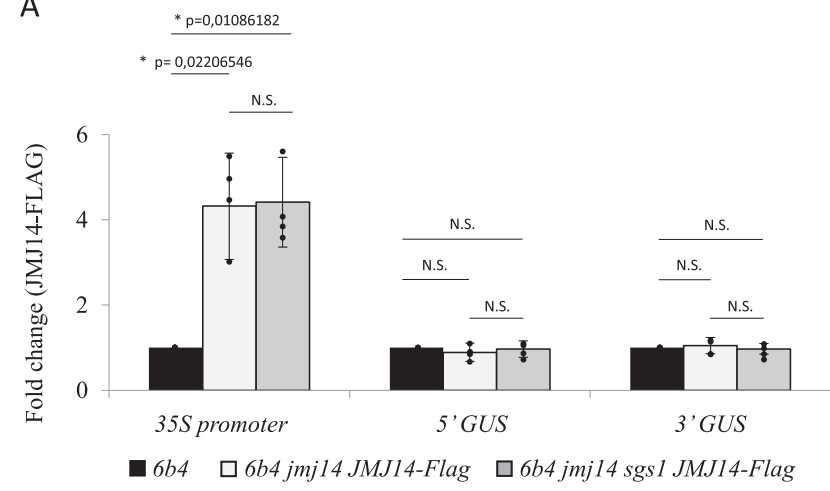

B

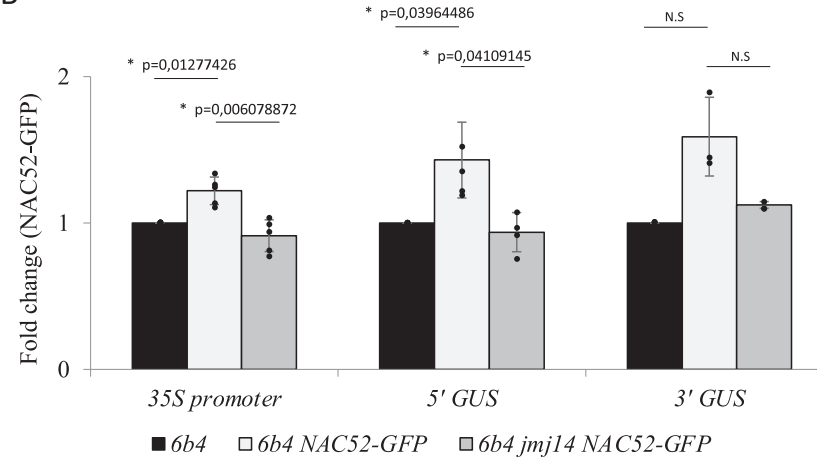

Fig. 7 Hierarchical binding of JMJ14 and NAC52 to the promoter and coding sequence of the p35S:GUS transgene, respectively. A ChIP-qPCR analyses were performed on the aerial part of 17-day-old seedlings of the indicated genotypes using a Flag antibody to immunoprecipitate JMJ14Flag. Levels are given as percentages of IPsample/IP6b4 relative to GAPDH, which was used as a control. The graphical representation shows the fold change as the mean of four replicates. Error bars represent the standard deviation of these four replicates. The significance was assayed with a Bonferroni corrected two-sides $t$-test $(P<0.05)$. Results indicate a relative enrichment of JMJ14 on the 355 promoter of the 664 locus, which was not affected by the sgs1 mutation impairing NAC52. B ChIP-qPCR analyses were performed on the aerial part of 17-day-old seedlings of the indicated genotypes using a GFP antibody to immunoprecipitate NAC52-GFP. Levels are given as percentages of IPsample/IP6b4 relative to GAPDH, which was used as a control. The graphical representation shows the fold change as the mean of five replicates. Error bars represent the standard deviation of these five replicates. The significance was assayed with a Bonferroni corrected two-sides $t$-test $(P<0,05)$. Results indicate a relative enrichment of NAC52 on the 355 promoter and the beginning of the GUS coding sequence of the $6 b 4$ locus, which was abolished by the jmj14 mutation, indicating a hierarchical action of JMJ14 and NAC52 at the 664 locus.

hyper $\mathrm{CHH}$ methylation, while the only endogenous hyper $\mathrm{CHG}$ methylation found in nac50 nac52 showed hyper $\mathrm{CHH}$ methylation but not hyper CG methylation (Supplementary Data 1). Therefore, it is most likely that the endogenous DMRs observed between wild type and either jmj14 or nac50 nac52 correspond to endogenous genomic sequences that exhibit highly variable methylation levels ${ }^{36,37}$. Together, WGBS analysis of WT, jmj14 and nac50 nac52 plants indicates that JMJ14 and NAC50/ NAC52 specifically limit the level of DNA methylation of transgenes and have essentially no effect on the DNA methylation status of endogenous genes.

To further address the role of NAC50/NAC52 in PTGS, a pUBQ10:NAC52-GFP transgene was generated and introduced into the $664 \mathrm{WT}$ line. The $\mathrm{pUBQ} 10$ promoter drives constitutive expression of transgenes in Arabidopsis. At first, we tested whether the $p U B Q 10: N A C 52-G F P$ constructs expressed a functional protein that actually interacts with JMJ14. To do so, coimmunoprecipitation (co-IP) experiments were performed on plants resulting from a cross between a $P J M J 14: J M J 14-M y c$ line $e^{46}$ and the $664+p U B Q 10: N A C 52-G F P$ line. As expected, JMJ14Myc and NAC52-GFP proteins co-precipitated in pull-down assays using a GFP antibody (Supplementary Fig. 4). Furthermore, ChIP was conducted on the $664+p U B Q 10: N A C 52-G F P$ line using GFP antibodies, followed by $\mathrm{qPCR}$ using primer pairs located in the $35 S$ promoter, and in the $5^{\prime}$ and $3^{\prime}$ regions within the GUS transgene body. The ChIP-qPCR analysis revealed a significant enrichment of NAC52 in the $35 S$ promoter and the $5^{\prime}$ region of the GUS coding sequence (Fig. 7B).

Finally, to address the hierarchical action of JMJ14 and NAC50/NAC52 in PTGS, we generated $6 b 4$ jmj14 nac52+ pJMJ14:3xFlag-JMJ1443 and 664 jmj14 + pUBQ10:NAC52-GFP lines. ChIP-qPCR revealed that the association of JMJ14 with chromatin at the 35S:GUS transgene was not affected by the nac52(sgs1) mutation (Fig. 7A), whereas the association of NAC52 was impaired by the jmj14 mutation (Fig. 7B). Altogether, these results strongly suggest that JMJ14 binds to chromatin at the promoter of the $p 35 S: G U S$ transgene, which promotes the binding of NAC50/NAC52 to the $35 \mathrm{~S}$ promoter and the downstream GUS coding sequence. Our results are consistent with JMJ14 and NAC50/NAC52 acting in a chromatin complex to prevent $\mathrm{CHG}$ methylation throughout the entire transgene, thus allowing aberrant RNAs to be produced, which in turn induce PTGS.

IBM1 impairment slightly decreases CHH and CHG methylation in the transgene body and promotes PTGS. To test further the hypothesis that transgene methylation influences PTGS capacity, the $6 b 4$ locus was introduced into the $i b m 1$ mutant background. IBM1 encodes an $\mathrm{H} 3 \mathrm{~K} 9 \mathrm{me} 2$ demethylase that suppresses $\mathrm{CHH}$ and $\mathrm{CHG}$ methylation in the body of about half of the endogenous genes of Arabidopsis ${ }^{33-35,47}$. We therefore expected $6 b 4 \mathrm{ibm} 1$ plants to exhibit increased $\mathrm{CHH}$ and $\mathrm{CHG}$ methylation in the GUS coding sequence and suppress induction of PTGS when grafted onto silenced GUS rootstocks. However, while WGBS analysis confirmed increased $\mathrm{CHH}$ and $\mathrm{CHG}$ methylation in the body of 9000 endogenous genes (Fig. 6 and Supplementary Data 1), $\mathrm{CHH}$ and $\mathrm{CHG}$ methylation remained the same or slightly decreased in transgenic sequences in $6 b 4 \mathrm{ibm} 1$ plants (Fig. 5 and Supplementary Fig. 3B). The decrease in $\mathrm{CHG}$ methylation in transgenic sequences of $6 b 4 \mathrm{ibm} 1$ plants was most obvious in the GUS coding sequence (Fig. 5B). These results confirmed that actively expressed transgenes and endogenous genes exhibit major epigenetic differences. Moreover, spontaneous triggering of GUS PTGS occured in $20 \%$ of $6 b 4 \mathrm{ibm} 1$ plants (Table 1 and Supplementary Fig. 5), indicating that JMJ14 and IBM1 have opposite effects on transgene PTGS.

\section{Discussion}

Endogenous protein-coding genes produce aberrant RNAs that are efficiently degraded by RNA quality control (RQC) pathways. In plants, this is essential to prevent the conversion of aberrant RNAs into dsRNA by RDRs, which would result in the production of siRNAs that could destroy homologous and essential mRNAs. Indeed, RQC impairment causes lethality in Arabidopsis, which can be rescued by suppressing RDR6 activity ${ }^{21,25}$. Similarly, transgenes produce aberrant RNAs in proportion to the level of transcript, and provided the aberrant RNAs do not exceed an abundance threshold within the cell, they are also eliminated by RQC. However, if RQC is impaired or saturated by high levels 
of transcription, both transgenes and endogenous genes can be subjected to RDR6-dependent PTGS ${ }^{15,16}$. Occasionally, endogenous loci in plants can be subjected to PTGS despite a functioning RQC pathway being present. However, these loci generally exhibit genomic rearrangements involving gene duplication events that allow the production of dsRNA and induction of PTGS ${ }^{48-54}$. It is assumed that such rearrangements involving endogenous genes are tolerated because they only adversely affect dispensable genes. Obviously, transgenes are also dispensable because they correspond to sequences that do not exist naturally in plants, and the transgene loci that induce PTGS the most efficiently involve strong promoters and/or tandem insertion of multiple copies of the transgene, often in inverted orientations ${ }^{55}$. High rates of transcription from multiple transgenes increases the chances of aberrant RNA by-products forming, and only a fraction of this aberrant RNA needs to escape degradation by RQC to be recruited by RDR6, and converted into dsRNA to induce PTGS.

Despite these similarities, actively expressed transgenes are generally more susceptible to PTGS than endogenous genes. These differences between actively expressed transgenes and endogenous genes have long remained a mystery. Our finding that transgenes and endogenous genes exhibit different epigenetic characteristics is particularly interesting because transgenes are transferred into the host genome as naked DNA or naked DNA associated with Agrobacterium proteins, and once integrated into the chromosome, must associate with histones to form chromatin. At present, the type of eukaryotic histones that associate with naked or newly integrated foreign DNA is not known. One could imagine that the insertion of extrachromosomal DNA into the chromosome attracts particular histone marks to label this DNA as 'new or foreign'. It seems likely that what happens for transgenes also occurs when a new copy of a retrotransposon integrates into the genome. Supporting this hypothesis, it has been shown that activation of retrotransposons triggers a PTGS response, just like transgenes $\mathrm{do}^{56-58}$. Although this hypothesis is attractive, our findings suggest that there are striking epigenetic differences between transgenes and endogenous loci, including genes and stabilized transposons. Here, we showed that transgenes and endogenous loci (genes and stabilized transposons) behave differently when the histone H3K4me3 demethylase JMJ14, and two cooperating transcription factors that interact with JMJ14, NAC50, and NAC52, are impaired. Indeed, in jmj14 or nac50 nac52 mutants, an increase in CHG methylation, and $\mathrm{CHH}$ methylation to a lesser extent, is observed at the $6 b 4$ transgene locus (Fig. 5 and Supplementary Fig. 3). An increase in CHG methylation was particularly evident in the promoter and gene body of the p35S:GUS transgene, and also parts of the integrated Agrobacterium vector sequences (Fig. 5 and Supplementary Fig. 3), whereas no change in DNA methylation profiles was observed at any endogenous genomic loci in these mutants (Fig. 6 and Supplementary Data 1). Remarkably, the jmj14 and nac50nac52 mutations also suppress the triggering of PTGS of the p35S:GUS and $p 35 S: G F P$ when these transgenic mutant lines were used as scions grafted onto rootstocks showing PTGS (Fig. 4 and Table 1). Moreover, $\mathrm{CHH}$ and $\mathrm{CHG}$ hypermethylation, and not simply the absence of JMJ14 or NAC50/NAC52, appears causal in preventing PTGS because $6 b 4 \mathrm{jmj} 14 \mathrm{drm} 2 \mathrm{~cm}+3$ scions trigger PTGS when grafted onto silenced rootstocks (Table 1), indicating that the impairment of DRM2-CMT3-dependent CHH and CHG methylation in a jmj14 background restores the susceptibility of transgenes to graft-transmissible PTGS. As JMJ14 and NAC52 bind to the p35S:GUS transgene within the 664 locus (Fig. 7), we propose that the interacting complex limits $\mathrm{CHG}$ and $\mathrm{CHH}$ methylation of the GUS coding sequence, allowing the production of aberrant RNAs that are required to trigger and maintain PTGS.
Supporting this hypothesis, the $6 b 4 \mathrm{drm} 2 \mathrm{cmt} 3$ transgenic line lacks $\mathrm{CHH}$ and $\mathrm{CHG}$ methylation of the GUS coding sequence and triggers PTGS spontaneously, whereas the $664 \mathrm{WT}$ line exhibits a low $\mathrm{CHH}$ and $\mathrm{CHG}$ methylation level of the GUS coding sequence that is nevertheless sufficient to block the spontaneous triggering of PTGS.

The $6 b 4$ T-DNA insertion locus contains not only an intact TDNA but also the entire Agrobacterium binary vector sequence alongside the left border of the T-DNA (Supplementary Fig. 2). Remarkably, the $\mathrm{CHG}$ and $\mathrm{CHH}$ methylation profiles of the vector sequences were similar to the $p 35 S$ :GUS transgene in WT plants and increased in the jmj14 mutant in a DRM2-CMT3dependent manner (Supplementary Fig. 3). These results suggest that not just promoter-driven transgenes, but also promoterless foreign DNA sequences could be subject to JMJ14-dependent suppression of $\mathrm{CHG}$ and $\mathrm{CHH}$ methylation, resulting in the production of aberrant RNA and potential PTGS of homologous mRNA. It is interesting to note that bombardment of transgenic tobacco expressing a $p 35 S$ :GFP transgene with promoterless GFP DNA sequences can induce PTGS 59 . Adding to this earlier report, our data are consistent with promoter-independent aberrant RNA transcripts being produced from foreign DNA sequences prior to or immediately after integration into the plant chromosome. In contrast to the p35S:GUS transgene and Agrobacterium vector sequences, $\mathrm{CHG}$ and $\mathrm{CHH}$ methylation of the pNOS: NPTII:tNOS transgene within the 664 locus remained low in the jmj14 and nac50 nac52 mutant backgrounds (Supplementary Fig. 3). This could be explained by JMJ14 not being able to bind to the Agrobacterium NOS promoter, which may have been selected for during the evolution of Agrobacterium as a pathogen to ensure that the transferred oncogenes on the T-DNA were not epigenetically distinguishable from endogenous plant genes.

$\mathrm{H} 3 \mathrm{~K} 4$ trimethylation and DNA methylation are considered to have antagonistic influences activating and repressing expression of endogenous genes, respectively ${ }^{46}$. Therefore, it was expected that for endogenous loci in jmj14 mutants, where H3K4me3 levels increase, DNA methylation would decrease. However, our WGBS analysis revealed that there were minimal changes in DNA methylation levels within endogenous genes of jmj14 (Fig. 6), further emphasizing the difference between transgenes and endogenous sequences. Nevertheless, we asked whether the binding of JMJ14 could promote the production of aberrant RNAs from endogenous genes and their subsequent transformation into siRNAs, like it does at the p35S:GUS transgene of the $6 b 4$ locus. If this was the case, one would expect that the fraction of endogenous genes that produce siRNAs when RQC is impaired $^{21,25,60}$ would be enriched for genes that bind JMJ14 ${ }^{45}$. However, the fraction of siRNA-producing endogenous genes that bind JMJ14 is exactly that expected by chance (Supplementary Fig. 6), whether considering the siRNA-producing endogenous genes identified in the decapping mutants $d c p 2$ and $v s^{21}$, the siRNA-producing endogenous genes identified in the exonuclease double mutant $x r n 4 s k i 2^{25}$, or the siRNA-producing endogenous genes identified when plants are infected by viruses that inhibit RQC 60 . Therefore, unlike the p35S:GUS transgene of the 664 locus, which binds JMJ14, endogenous genes that bind JMJ14 do not appear particularly prone to producing endogenous siRNAs when RQC is compromised. Altogether with our results, these results reinforce the idea that transgene DNA hypermethylation caused by the absence of JMJ14 is specific to transgenes and not endogenous genes.

We not only found that transgenes and endogenous genes exhibit different epigenetic behavior with regard to the H3K4me3 demethylase JMJ14, but also to the histone H3K9me2 demethylase IBM1 ${ }^{33-35,47}$. Indeed, in jmj14 mutant plants, an increase in DNA methylation is observed in the newly inserted sequences of 
the $6 b 4$ locus but not in endogenous genes, whereas in $i b m 1$ mutant plants, an increase in DNA methylation is observed in endogenous genes but not in the newly inserted sequences of the $6 b 4$ locus (Figs. 5 and 6 and Supplementary Fig. 3). Moreover, whereas $6 b 4$ plants do not trigger PTGS spontaneously but undergo systemic PTGS when grafted on silenced rootstocks, 664 jmj14 plants do not trigger PTGS spontaneously and are incapable of undergoing systemic PTGS, while $6 b 4 \mathrm{ibm} 1$ plants trigger PTGS spontaneously, similar to $6 b 4 \mathrm{drm} 2 \mathrm{cmt} 3$ plants (Table 1 and Supplementary Fig. 5), indicating that JMJ14 and IBM1 have opposite effects on transgene PTGS. It may be assumed that actively expressed transgenes behave epigenetically the same as endogenous genes. However, our data suggest that despite being stably expressed in a WT background, transgenes exhibit epigenetic features that strongly differs from that of endogenous genes with regard to JMJ14 and IBM1 regulation.

To conclude, our research shows that JMJ14 maintains expressed transgenes in an epigenetic state that allows a residual level of aberrant RNA to be produced from the transgene body, thereby rendering the transgene particularly susceptible to PTGS. This epigenetic phenomenon could therefore represent an evolutionary probation period for the expressed transgene, until it is either completely silenced, or alternatively, epigenetically accepted as an endogenous locus in the new host genome.

\section{Methods}

Plant material. The transgenic reporter lines 10027-3, 214, L1, $6 b 4$ and 6b4-306, and the mutants jmj14-4, nac52sgs1, nac50 nac52, rdr6 sgs2-1, cmt3-7, drm2-3, ibm11 , vcs-9 all are in the Arabidopsis accession Columbia $13,21,26,38,40,44,47,61,62$. The transgenic lines carrying the tagged constructs pJMJ14:JMJ14-Myc, pJMJ14:FlagJMJ14 and $P U B Q 10: N A C 52-G F P$ also are in the Arabidopsis accession Columbia $28,43,46$. Primers used for genotyping are listed in Supplementary Table 1.

\section{Growth conditions and grafting techniques. Arabidopsis seeds were surface-} sterilized, sown on a nutritive medium (1.3\% S-medium Duchefa, $1 \%$ Phytoblend agar), vernalized at $4{ }^{\circ} \mathrm{C}$ for 2 days, and then placed in a culture chamber at $23^{\circ} \mathrm{C}$, $70 \%$ humidity, $120 \mu \mathrm{E} \mathrm{m}^{-2}$ light with a $16 \mathrm{~h}$ light $/ 8 \mathrm{~h}$ dark (long-days) or $8 \mathrm{~h}$ light/ $16 \mathrm{~h}$ dark (short-days) photoperiod. Seedlings grown under long-day conditions were transferred to soil after 2 weeks. Seedlings grown under short-day conditions were used for grafting expertiments as described in ref. ${ }^{63}$. Briefly, 6 days after gemination, seedlings were cut transversely across the hypocotyl using a razor blade $\left(90^{\circ}\right.$ butt graft). Then, scions and rootstocks were placed on a nitrocellulose filter (Hybond). Hypocotyls of scions and rootstock were introduced into a silicon microtube ( $2 \mathrm{~mm}$ long) to connect them to each other, and incubated under shortday conditions ( $8 \mathrm{~h}$ light, $16 \mathrm{~h}$ dark) for 7 to 14 days. Grafted seedlings that did not show adventitious roots were transferred to soil and grown under a short-days photoperiod.

GUS activity and GUS RNA analysis. GUS protein was extracted from plant leaves, and GUS activity was quantified by monitoring the quantity of 4methylumbelliferone produced by cleavage of the substrate 4-methylumbelliferylb-D-glucuronide (Duchefa) on a fluorometer (Thermo Scientific fluoroskan ascent) ${ }^{64}$.

RNA extraction and HMW or LMW RNA gel blot analyses were performed using $5-10 \mu \mathrm{g}$ of total RNA and GUS, U6 and $25 S$ probes $^{16}$. For the reverse transcription, RNA was treated with DNaseI (Invitrogen) and $1 \mu \mathrm{g}$ of DNA-free RNA was reverse transcribed with the primer called RT_ASGUS_Linker using the RevertAid H Minus Reverse Transcriptase (ThermoFisher, http://www. thermofisher.com/) Amplification was performed by using the LK and RbcS1Rev primers $^{11}$, and qPCR results were normalized with EiF1a ${ }^{11}$.

\section{Whole-genome bisulfite sequencing and DNA methylation analysis. Plants} were grown under short-day conditions for five weeks (experiment 1: 6b4-1, 6b4 jmj14, $6 b 4 \mathrm{jmj} 14 \mathrm{cmt} 3 \mathrm{drm} 2,6 \mathrm{~b} 4 \mathrm{cmt} 3 \mathrm{drm} 2$ ) or eight weeks (experiment 2: 6b4-2, $6 b 4$ nac50 nac52, $6 b 4 \mathrm{ibm} 1$ ). Genomic DNA was isolated from leaf tissue from a mix of eight plants per genotype using the Nucleospin Plant II Maxi kit (Macharey Nagel), and library preparation and sequencing was performed by BGI Genomics (Hong Kong). Briefly, genomic DNA was fragmented to 100-300 bp by sonication, end-repaired, and ligated to methylated adaptors. Bisulfite treatment was then performed using the EZ DNA Methylation-Gold kit (Zymo), and the bisulfitetreated fragments were PCR amplified and sequenced as paired-end $100 \mathrm{bp}$ reads (PE100) with DNBSEQ technology. Pre-processed high-quality reads were mapped to the TAIR10 genome using bismark with default settings for paired-end libraries ${ }^{65}$, and all downstream analysis were performed using custom R scripts. All figures presenting bisulfite data were generated using $\mathrm{R}$ version 4.0.2. A summary of all bisulfite sequencing data generated in this study is presented in Supplementary Table 2, and is accessible through NCBI's Gene Expression Omnibus (GSE152584).

ChIP-qPCR analysis. ChiP was performed on chromatin using $2 \mathrm{~g}$ of crosslinked in vitro shoots from 15-day-old seedlings ${ }^{66}$. Chromatin was sonicated ( $30 \mathrm{~s}$ "ON"/ $30 \mathrm{~s}$ "OFF") with a Bioruptor UCD200 (Diagenode). The chromatin solution was diluted 10 fold with ChiP dilution buffer. Fifty microliters of Dynabeads Protein G (invitrogen) and $25 \mu \mathrm{L}$ of GFP-Trap Dynabeads (chromotek) was washed twice with ChiP dilution buffer. Nineteen micrograms of Flag (Sigma F3165) antibodies were added to $\mathrm{G}$ Protein and incubated at least $2 \mathrm{~h}$ at $4{ }^{\circ} \mathrm{C}$ with gentle rotation. Beads were washed three times with ChiP dilution buffer. Then, $1 \mathrm{~mL}$ of the diluted chromatin was added to the beads/antibodies and incubated overnight at $4{ }^{\circ} \mathrm{C}$ with gentle agitation. Beads were washed as decribed ${ }^{66}$. After the last TE wash, reverse crosslinking (at least $4 \mathrm{~h}$ at $65^{\circ} \mathrm{C}$ ) and elution were performed using an IPure kit (Diagenode (AL-100-0100)). The final elution was performed in $60 \mu \mathrm{L}$ and the chromatin was stored at $-20^{\circ} \mathrm{C}$ until analysis.

Using the Biorad-CFX-Maestro Software, the ChIP was analyzed by qPCR using $2 \mu \mathrm{L}$ of chromatin in triplicate. Primers are listed in Supplementary Table 1. The mean of three qPCR results (with $\mathrm{SD}<0.4$ cycle threshold) was used for each point. Glyceraldehyde-3-phosphate dehydrogenase (GAPDH)) was used as an internal ref. ${ }^{67}$. Results are represented as fold change: normalized expression $(\Delta \Delta \mathrm{Cq})$ given by the ratio of Relative Quantity of the sample (2(Cq 6b4-Cq sample) for each identical oligo with $100 \%$ efficiency) divided by the Relative Quantity of GAPDH. At least two biological replicates were analyzed each time. Results show the mean and SD of the independent biological replicates.

Co-immunoprecipitation experiment. For each bulk of plants 15 days after germination, $2 \mathrm{~g}$ of fresh tissue was ground in CoIP Buffer $(50 \mathrm{mM}$ Tris $\mathrm{pH} 7.5$, $100 \mathrm{mM}$ EDTA, 15\% Glycerol, 1\% NP40, 1\% Triton X100, 1x cOmplete ${ }^{\mathrm{TM}}$ Protease Inhibitor Cocktail (Merck). After $30 \mathrm{~min}$ on ice, samples were centrifugated at $7650 \times g$ at $4{ }^{\circ} \mathrm{C}$ and the supernatant quantified after miracloth filtration. Five micrograms of this crude extract was submitted to preclear on $50 \mu \mathrm{L}$ of magnetic beads coated with protein A for $2 \mathrm{~h}$ at $4{ }^{\circ} \mathrm{C}$. After magnetic decantation, $50 \mu \mathrm{L}$ of GFP-Trap_M (gtm-20, Chromotek) was rinsed (2x PBS0.1\% and 1x CoIP buffer) and added to the supernatant. This was incubated overnight at $4{ }^{\circ} \mathrm{C}$ with gentle agitation. The GFP beads were then rinsed $6 \mathrm{x}$ in CoIP buffer, and finally adjusted to $40 \mu \mathrm{L}$ of CoIP buffer, $5 \mu \mathrm{L}$ of $6 \mathrm{x}$ Laemmli buffer and incubated at $95^{\circ} \mathrm{C}$ for $5 \mathrm{~min}$. After vortexing, $40 \mu \mathrm{L}$ of this final supernatant was loaded onto a $10 \%$ polyacrylamide gel. Following sodium dodecyl sulphate-polyacrylamide gel electrophoresis, western blotting was performed using anti myc (mouse 9E10, sigma-M4439), anti GFP (Rabbit Abcam-Ab290) or anti tubulin (mouse B-5-1-2, sigma-T5168) antibodies. An ImageQuant-LAS4000 (GE Healthcare) was used for detection.

Reporting summary. Further information on research design is available in the Nature Research Reporting Summary linked to this article.

\section{Data availability}

Data supporting the findings of this work are available within the paper and its Supplementary Information files. A reporting summary for this Article is available as a Supplementary Information file. The datasets and plant materials generated and analyzed during the current study are available from the corresponding author upon request. Bisulfite sequencing data are accessible through NCBI's Gene Expression Omnibus GSE152584. The source data underlying Figs. 1A-C, 2B, and 4C, as well as Supplementary Figs. 4 and 5 are provided as a Source Data file. Source data are provided with this paper.

Received: 21 September 2020; Accepted: 6 April 2021; Published online: 13 May 2021

\section{References}

1. Bartel, D. P. MicroRNAs: genomics, biogenesis, mechanism, and function. Cell 116, 281-297 (2004).

2. Baulcombe, D. RNA silencing in plants. Nature 431, 356-363 (2004).

3. Lippman, Z. \& Martienssen, R. The role of RNA interference in heterochromatic silencing. Nature 431, 364-370 (2004).

4. Havecker, E. R. et al. The Arabidopsis RNA-directed DNA methylation argonautes functionally diverge based on their expression and interaction with target loci. Plant Cell 22, 321-334 (2010).

5. Xie, Z. et al. Genetic and functional diversification of small RNA pathways in plants. PLoS Biol. 2, 642-652 (2004) 
6. Zhang, Z., Liu, X., Guo, X., Wang, X. J. \& Zhang, X. Arabidopsis AGO3 predominantly recruits 24 -nt small RNAs to regulate epigenetic silencing. Nat. Plants 2, 16049 (2016).

7. Law, J. A. \& Jacobsen, S. E. Establishing, maintaining and modifying DNA methylation patterns in plants and animals. Nat. Rev. Genet. 11, 204-220 (2010).

8. Zemach, A. et al. The Arabidopsis nucleosome remodeler DDM1 allows DNA methyltransferases to access H1-containing heterochromatin. Cell 153, 193-205 (2013).

9. Martinez de Alba, A. E., Elvira-Matelot, E. \& Vaucheret, H. Gene silencing in plants: a diversity of pathways. Biochim. Biophys. Acta 1829, 1300-1308 (2013).

10. Mlotshwa, S. et al. DICER-LIKE2 plays a primary role in transitive silencing of transgenes in Arabidopsis. PLoS ONE 3, e1755 (2008).

11. Parent, J. S. et al. Post-transcriptional gene silencing triggered by sense transgenes involves uncapped antisense RNA and differs from silencing intentionally triggered by antisense transgenes. Nucleic Acids Res. 43, 8464-8475 (2015).

12. Vaistij, F. E., Jones, L. \& Baulcombe, D. C. Spreading of RNA targeting and DNA methylation in RNA silencing requires transcription of the target gene and a putative RNA-dependent RNA polymerase. Plant Cell 14, 857-867 (2002).

13. Brosnan, C. A. et al. Nuclear gene silencing directs reception of long-distance mRNA silencing in Arabidopsis. Proc. Natl Acad. Sci. USA 104, 14741-14746 (2007).

14. Palauqui, J. C., Elmayan, T., Pollien, J. M. \& Vaucheret, H. Systemic acquired silencing: transgene-specific post-transcriptional silencing is transmitted by grafting from silenced stocks to non-silenced scions. EMBO J. 16, 4738-4745 (1997).

15. Gazzani, S., Lawrenson, T., Woodward, C., Headon, D. \& Sablowski, R. A link between mRNA turnover and RNA interference in Arabidopsis. Science 306, 1046-1048 (2004).

16. Gy, I. et al. Arabidopsis FIERY1, XRN2, and XRN3 are endogenous RNA silencing suppressors. Plant Cell 19, 3451-3461 (2007).

17. Luo, Z. \& Chen, Z. Improperly terminated, unpolyadenylated mRNA of sense transgenes is targeted by RDR6-mediated RNA silencing in Arabidopsis. Plant Cell 19, 943-958 (2007).

18. Goeres, D. C. et al. Components of the Arabidopsis mRNA decapping complex are required for early seedling development. Plant Cell 19, 1549-1564 (2007).

19. Chen, C. Y. \& Shyu, A. B. Mechanisms of deadenylation-dependent decay. Wiley Interdiscip. Rev. RNA 2, 167-183 (2011).

20. Gregory, B. D. et al. A link between RNA metabolism and silencing affecting Arabidopsis development. Dev. Cell 14, 854-866 (2008).

21. Martinez de Alba, A. E. et al. In plants, decapping prevents RDR6-dependent production of small interfering RNAs from endogenous mRNAs. Nucleic Acids Res. 43, 2902-2913 (2015).

22. Moreno, A. B. et al. Cytoplasmic and nuclear quality control and turnover of single-stranded RNA modulate post-transcriptional gene silencing in plants. Nucleic Acids Res. 41, 4699-4708 (2013).

23. Thran, M., Link, K. \& Sonnewald, U. The Arabidopsis DCP2 gene is required for proper mRNA turnover and prevents transgene silencing in Arabidopsis. Plant J. 72, 368-377 (2012).

24. $\mathrm{Yu}, \mathrm{A}$. et al. Second-site mutagenesis of a hypomorphic argonaute1 allele identifies SUPERKILLER3 as an endogenous suppressor of transgene posttranscriptional gene silencing. Plant Physiol. 169, 1266-1274 (2015).

25. Zhang, X. et al. Plant biology. Suppression of endogenous gene silencing by bidirectional cytoplasmic RNA decay in Arabidopsis. Science 348, 120-123 (2015).

26. Le Masson, I. et al. Mutations in the Arabidopsis $\mathrm{H} 3 \mathrm{~K} 4 \mathrm{me} 2 / 3$ demethylase JMJ14 suppress posttranscriptional gene silencing by decreasing transgene transcription. Plant Cell 24, 3603-3612 (2012).

27. Searle, I. R., Pontes, O., Melnyk, C. W., Smith, L. M. \& Baulcombe, D. C. JMJ14, a JmjC domain protein, is required for RNA silencing and cell-to-cell movement of an RNA silencing signal in Arabidopsis. Genes Dev. 24, 986-991 (2010).

28. Butel, N., Le Masson, I., Bouteiller, N., Vaucheret, H. \& Elmayan, T. sgs1: a neomorphic nac52 allele impairing post-transcriptional gene silencing through SGS3 downregulation. Plant J. 90, 505-519 (2017).

29. Rountree, M. R. \& Selker, E. U. DNA methylation inhibits elongation but not initiation of transcription in Neurospora crassa. Genes Dev. 11, 2383-2395 (1997).

30. Bewick, A. J. \& Schmitz, R. J. Gene body DNA methylation in plants. Curr. Opin. Plant Biol. 36, 103-110 (2017).

31. Choi, J., Lyons, D. B., Kim, M. Y., Moore, J. D. \& Zilberman, D. DNA methylation and histone $\mathrm{H} 1$ jointly repress transposable elements and aberrant intragenic transcripts. Mol. Cell 77, 310-323 (2020). e7.
32. Neri, F. et al. Intragenic DNA methylation prevents spurious transcription initiation. Nature 543, 72-77 (2017)

33. Inagaki, S. et al. Autocatalytic differentiation of epigenetic modifications within the Arabidopsis genome. EMBO J. 29, 3496-3506 (2010).

34. Miura, A. et al. An Arabidopsis jmjC domain protein protects transcribed genes from DNA methylation at CHG sites. EMBO J. 28, 1078-1086 (2009)

35. Wang, $\mathrm{X}$. et al. RNA-binding protein regulates plant DNA methylation by controlling mRNA processing at the intronic heterochromatin-containing gene IBM1. Proc. Natl Acad. Sci. USA 110, 15467-15472 (2013).

36. Becker, C. et al. Spontaneous epigenetic variation in the Arabidopsis thaliana methylome. Nature 480, 245-249 (2011).

37. Schmitz, R. J. et al. Transgenerational epigenetic instability is a source of novel methylation variants. Science 334, 369-373 (2011).

38. Taochy, C. et al. A genetic screen for impaired systemic RNAi highlights the crucial role of DICER-LIKE 2. Plant Physiol. 175, 1424-1437 (2017).

39. Cao, J. et al. Can-Seq: a PCR and DNA sequencing strategy for identifying new alleles of known and candidate genes. Plant Methods 16, 16 (2020).

40. Beclin, C., Boutet, S., Waterhouse, P. \& Vaucheret, H. A branched pathway for transgene-induced RNA silencing in plants. Curr. Biol. 12, 684-688 (2002).

41. Parent, J. S., Bouteiller, N., Elmayan, T. \& Vaucheret, H. Respective contributions of Arabidopsis DCL2 and DCL4 to RNA silencing. Plant J. 81, 223-232 (2015).

42. Gelvin, S. B. Integration of Agrobacterium T-DNA into the Plant Genome. Annu. Rev. Genet. 51, 195-217 (2017).

43. Jeong, J. H. et al. Repression of FLOWERING LOCUS T chromatin by functionally redundant histone $\mathrm{H} 3$ lysine 4 demethylases in Arabidopsis. PLoS ONE 4, e8033 (2009)

44. Ning, Y. Q. et al. Two novel NAC transcription factors regulate gene expression and flowering time by associating with the histone demethylase JMJ14. Nucleic Acids Res. 43, 1469-1484 (2015).

45. Zhang, S. et al. C-terminal domains of a histone demethylase interact with a pair of transcription factors and mediate specific chromatin association. Cell Discov. 1, 15003 (2015).

46. Deleris, A. et al. Involvement of a Jumonji-C domain-containing histone demethylase in DRM2-mediated maintenance of DNA methylation. EMBO Rep. 11, 950-955 (2010).

47. Saze, H., Shiraishi, A., Miura, A. \& Kakutani, T. Control of genic DNA methylation by a jmjC domain-containing protein in Arabidopsis thaliana. Science 319, 462-465 (2008).

48. Clough, S. J. et al. Features of a $103-\mathrm{kb}$ gene-rich region in soybean include an inverted perfect repeat cluster of CHS genes comprising the I locus. Genome 47, 819-831 (2004).

49. Coen, E. S. \& Carpenter, R. A semi-dominant allele, niv-525, acts in trans to inhibit expression of its wild-type homologue in Antirrhinum majus. EMBO J. 7, 877-883 (1988)

50. Della Vedova, C. B. et al. The dominant inhibitory chalcone synthase allele C2-Idf (inhibitor diffuse) from Zea mays (L.) acts via an endogenous RNA silencing mechanism. Genetics 170, 1989-2002 (2005).

51. Durand, S., Bouche, N., Perez Strand, E., Loudet, O. \& Camilleri, C. Rapid establishment of genetic incompatibility through natural epigenetic variation. Curr. Biol. 22, 326-331 (2012).

52. Melquist, S. \& Bender, J. Transcription from an upstream promoter controls methylation signaling from an inverted repeat of endogenous genes in Arabidopsis. Genes Dev. 17, 2036-2047 (2003).

53. Tuteja, J. H., Clough, S. J., Chan, W. C. \& Vodkin, L. O. Tissue-specific gene silencing mediated by a naturally occurring chalcone synthase gene cluster in Glycine max. Plant Cell 16, 819-835 (2004).

54. Tuteja, J. H., Zabala, G., Varala, K., Hudson, M. \& Vodkin, L. O. Endogenous, tissue-specific short interfering RNAs silence the chalcone synthase gene family in glycine max seed coats. Plant Cell 21, 3063-3077 (2009).

55. Sidorenko, L. V. et al. GC-rich coding sequences reduce transposon-like, small RNA-mediated transgene silencing. Nat. Plants 3, 875-884 (2017).

56. Creasey, K. M. et al. miRNAs trigger widespread epigenetically activated siRNAs from transposons in Arabidopsis. Nature 508, 411-415 (2014).

57. Mari-Ordonez, A. et al. Reconstructing de novo silencing of an active plant retrotransposon. Nat. Genet. 45, 1029-1039 (2013).

58. Nuthikattu, S. et al. The initiation of epigenetic silencing of active transposable elements is triggered by RDR6 and 21-22 nucleotide small interfering RNAs. Plant Physiol. 162, 116-131 (2013).

59. Voinnet, O., Vain, P., Angell, S. \& Baulcombe, D. C. Systemic spread of sequence-specific transgene RNA degradation in plants is initiated by localized introduction of ectopic promoterless DNA. Cell 95, 177-187 (1998).

60. Cao, M. et al. Virus infection triggers widespread silencing of host genes by a distinct class of endogenous siRNAs in Arabidopsis. Proc. Natl Acad. Sci. USA 111, 14613-14618 (2014).

61. Elmayan, T. et al. Arabidopsis mutants impaired in cosuppression. Plant Cell 10, 1747-1758 (1998) 
62. Lindroth, A. M. et al. Requirement of CHROMOMETHYLASE3 for maintenance of CpXpG methylation. Science 292, 2077-2080 (2001).

63. Turnbull, C. G., Booker, J. P. \& Leyser, H. M. Micrografting techniques for testing long-distance signalling in Arabidopsis. Plant J. 32, 255-262 (2002).

64. Elmayan, T. \& Vaucheret, H. Expression of single copies of a strongly expressed $35 \mathrm{~S}$ transgene can be silenced post-transcriptionally. Plant J. $\mathbf{9}$, 787-797 (1996).

65. Krueger, F. \& Andrews, S. R. Bismark: a flexible aligner and methylation caller for Bisulfite-Seq applications. Bioinformatics 27, 1571-1572 (2011).

66. Gendrel, A. V., Lippman, Z., Martienssen, R. \& Colot, V. Profiling histone modification patterns in plants using genomic tiling microarrays. Nat. Methods 2, 213-218 (2005).

67. Czechowski, T., Stitt, M., Altmann, T., Udvardi, M. K. \& Scheible, W. R. Genome-wide identification and testing of superior reference genes for transcript normalization in Arabidopsis. Plant Physiol. 139, 5-17 (2005).

\section{Acknowledgements}

We thank Dr. Angélique Déléris for providing the pJMJ14:JMJ14-Myc line, Dr. Yoo-Sun Noh for providing the pJMJ14:Flag-JMJ14 line, and Dr. Xin-Jian He for providing the nac50 nac52 mutant.

\section{Author contributions}

N.B., A.Y., I.L.M., C.T., N.R.G., J.C., and S.B. conducted the experiments; F.B., T.E., A.S., B.J.C., and H.V. analyzed the data; B.J.C. and H.V. designed the experiments and wrote the paper.

\section{Competing interests}

The authors declare no competing interests.

\section{Additional information}

Supplementary information The online version contains supplementary material available at https://doi.org/10.1038/s41467-021-22995-3.

Correspondence and requests for materials should be addressed to B.J.C. or H.V.

Peer review information Nature Communications thanks Brian Gregory and other, anonymous, reviewers for their contributions to the peer review of this work. Peer review reports are available.

Reprints and permission information is available at http://www.nature.com/reprints

Publisher's note Springer Nature remains neutral with regard to jurisdictional claims in published maps and institutional affiliations.

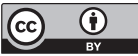

Open Access This article is licensed under a Creative Commons Attribution 4.0 International License, which permits use, sharing, adaptation, distribution and reproduction in any medium or format, as long as you give appropriate credit to the original author(s) and the source, provide a link to the Creative Commons license, and indicate if changes were made. The images or other third party material in this article are included in the article's Creative Commons license, unless indicated otherwise in a credit line to the material. If material is not included in the article's Creative Commons license and your intended use is not permitted by statutory regulation or exceeds the permitted use, you will need to obtain permission directly from the copyright holder. To view a copy of this license, visit http://creativecommons.org/ licenses/by/4.0/.

(C) The Author(s) 2021 